\title{
Influence of Design Parameters on the Short-Circuit Ruggedness of SiC Power MOSFETs
}

\author{
G. Romano, M. Riccio, L. Maresca, \\ G. Breglio, A. Irace \\ Department of Electrical Engineering and \\ Information Technologies \\ University of Naples Federico II \\ Naples, Italy \\ e-mail: gianpaolo.romano@unina.it
}

\author{
A. Fayyaz, A. Castellazzi \\ Power Electronics, Machines and \\ Control Group \\ University of Nottingham \\ United Kingdom
}

\begin{abstract}
This work aims to present an investigation on short-circuit (SC) failure behaviour of SiC Power MOSFETs due to the onset of thermal runaway. As inferable from experimental outcomes, it is related to the formation of hotspot, whose exact location is mainly unpredictable and dictated by device structure and design parameters non-uniformities. TCAD simulations were performed to examine the impact of some parameters mismatch on hotspot formation and failure occurrence.
\end{abstract}

Keywords - Silicon Carbide (SiC) Power MOSFET; Shortcircuit failure; Short-Circuit ruggedness; Thermal Runaway; hotspot; TCAD 2D simulation

\section{INTRODUCTION}

After the advent and the spread of Silicon Carbide (SiC) Power devices, especially Power MOSFETs [1], there has been an increasing interest in their failure mechanisms. Commercial devices should fulfil some robustness requirements, since in many applications stressful and out-of-SOA conditions might occur, even not rarely. Among all, short-circuit (SC) withstanding capability is a demanding feature for the design of reliable systems, and usually a device is required to survive a $10 \mu \mathrm{s}$ SC occurrence. A clear discernment on the failure origin could allow future devices to be more robust, increasing circuits reliability in case of undesired working conditions.

In recent years several works appeared, investigating and characterizing SiC Power MOSFETs. Many analyses reported $\mathrm{SC}$ devices behaviour, showing both single pulse and repetitive testing (e.g. [2]-[4]) highlighting maximum SC limits in different conditions, even spotting electro-thermal instability [5]. An analytical analysis of short-circuit capability has been proposed in [6] while two different failure modes have been identified and explained in [7]. Moreover, various physic-based compact models have been proposed, some including crucial electro-thermal effects (e.g. [8]-[9]).

In this paper, numerical simulations were the basis for the evaluation of design parameters impact on devices short-circuit capability, and their influence in creating weak spots. A TCAD structure was calibrated on measured transfer characteristics of a $1.2 \mathrm{kV}$ commercial Power MOSFET, nonetheless it does not reproduce the actual device geometry, and then it can be considered as a more general case study.

\section{ANALYSIS}

First, it is worth briefly recalling the results described in [7]. During single pulse test, temperature was suggested to be origin of two separate failure events. Moderate temperature increase (in case of relatively low power applied) could provoke a permanent damage on the top layers (resulting in gate/source terminals short). Consequently, it is impossible for the device to conduct anymore. The second case occurs for a large temperature increment. The amount of extra carriers thermally generated increases dramatically up to a value for which the leakage current is high enough to punch through the body/drift junction until it sets on thermal runaway phenomenon. If triggered, this positive feedback process makes the current focalize in a limited area. Fig.1 depicts the described situation observed experimentally, where the normalized temperature distribution at the turn-off $\left(\mathrm{t}_{\mathrm{PULSE}}=8 \mu \mathrm{s}\right)$ is shown. It was obtained, being synchronized exactly at the turn-off instant, using a custom IR thermography system [10], that allowed to clearly catch the hot spot just before the failure event.

It has to be noted that, in this case, the failure occurs approximately $1 \mu$ s after the turn-off (Fig.2). It is a failure mode usually observed in IGBTs [11], as well as current tails appearing at turn-off. This behaviour confirms that the failure cause could lie in a local increase of leakage current sustained by temperature.

During ON-state the current spreads nearly uniformly all over the device, but unavoidably some areas could have

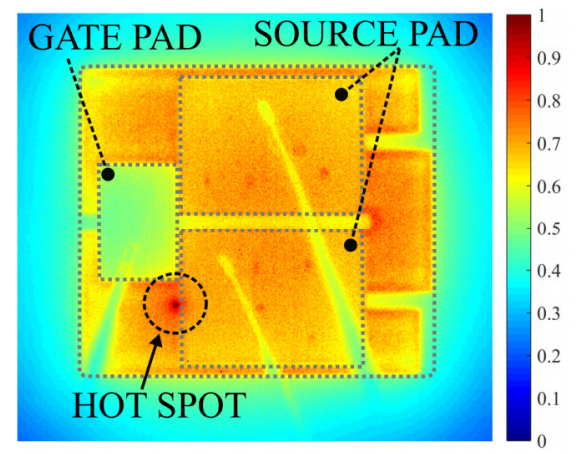

Fig. 1. Normalized temperature increase at $\mathrm{t}=8 \mu \mathrm{s}$ 


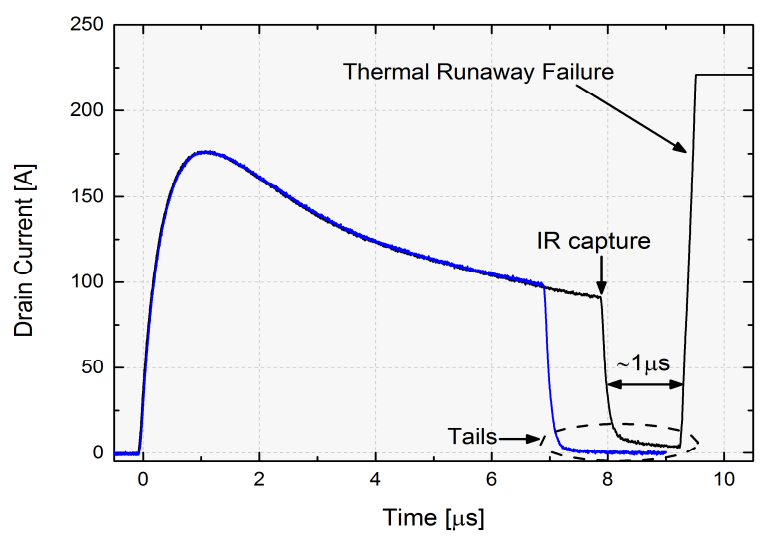

Fig. 2. Drain current short-circuit waveforms $\left(\mathrm{V}_{\mathrm{DS}}=600 \mathrm{~V} ; \mathrm{V}_{\mathrm{GS}}=18 \mathrm{~V} ; \mathrm{T}_{\mathrm{CASE}}=27^{\circ} \mathrm{C}\right)$

slightly higher current density than other. As an example, the current is moderately more condensed beneath and around the bonded wires, while outer areas present smaller current density. Therefore, temperature distribution is uniform over a large area, with minor gradients defined by top device structure (pads, metallization, bond wires, etc.). Inside that area, a cluster of cells could be weaker due to inevitably differences created during manufacturing processes. They could be prone to drain more current and eventually almost the all short-circuit current when thermal runaway occurs. Unfortunately, location where hotspot appears is impossible to predict.

Described situation was analysed thanks to electro-thermal (ET) physical simulations. The device was modelled by two parallel cells and was included in a mixed-mode schematic as depicted in Fig.3. The ratio of area factors of two cells was chosen such that:

$$
\begin{gathered}
A F_{1}+A F_{2}=A F_{T O T} \\
\frac{A F_{1}}{A F_{2}}=10^{4}
\end{gathered}
$$

CELL1 represents almost totally the device, while CELL2 models the cluster of cells where hotspot might originate, therefore taking into account the filament dimension usually very modest compared to the total area. Moreover, mismatch in some structure parameters was introduced in CELL2 to make it

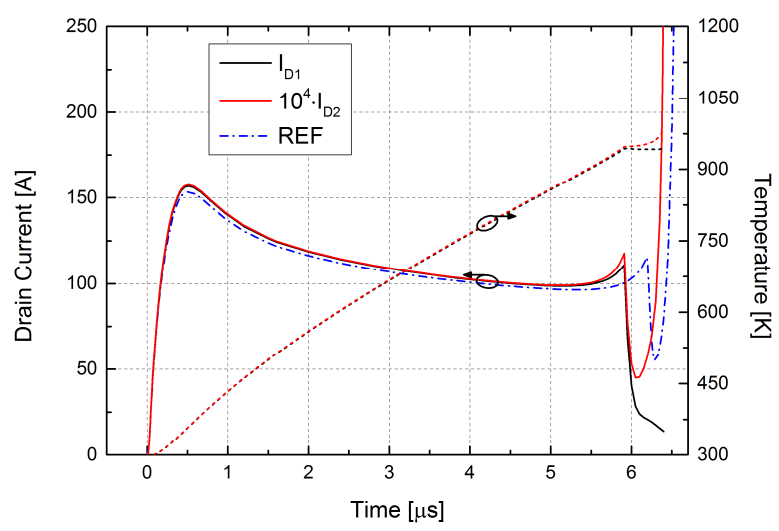

Fig.4. Simulated $\mathrm{I}_{\mathrm{D}} \mathrm{SC}$ waveforms - channel doping mismatch $\left(\mathrm{V}_{\mathrm{DS}}=800 \mathrm{~V} ; \mathrm{V}_{\mathrm{GS}}=18 \mathrm{~V} ; \mathrm{T}_{\mathrm{CASE}}=27^{\circ} \mathrm{C}\right)$

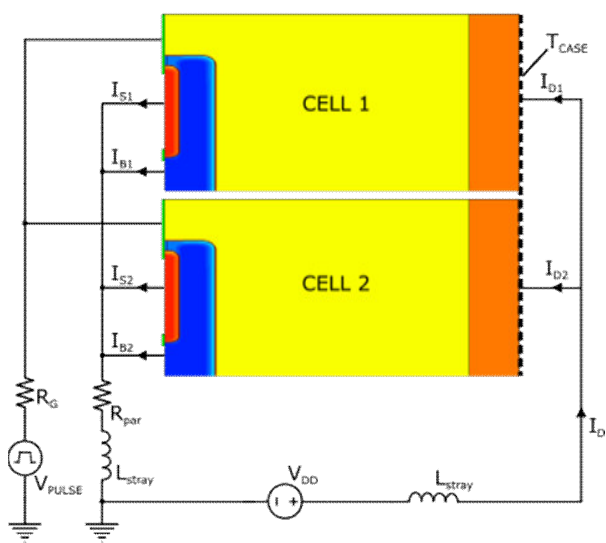

Fig. 3. Diagram of mixed-mode simulated circuit

"weak". A cell can be considered weak if, for any reason, it carries even slightly more current than other cells, hence being more likely to give rise to thermal runaway. It is thus expected that the short-circuit failure current would entirely flow in CELL2 if thermal runaway sets on. All the results are compared with a single cell structure calibrated on commercial device $\mathrm{I}_{\mathrm{D}}-\mathrm{V}_{\mathrm{GS}}$ curves, used as reference design.

\section{RESULTS}

Channel peak doping mismatch was first analysed, and results are reported in Fig.4. As the leakage current reaches a critical value, the short-circuit current moves from CELL1 to flow entirely through CELL2, until the device fails. It is visible from Fig.5 where current densities of both cells are reported. At the turn-off (Fig.5a), the current densities in both cells are almost balanced. Afterwards, the current in CELL1 starts to decrease, while is higher in CELL2 (Fig.5b). Finally, the weak cell carries all the failure current (Fig.5c) while CELL1 is completing its turn-off phase. It has to be noted that, as expected, the leakage current is partially formed by hole flowing through the body terminal. The reference cell is able to sustain about 280ns longer SC pulse.

Channel peak doping fluctuation is extremely dependent on technology process, but in this case, a barely $0.75 \%$ difference is enough to create a weak cell reducing maximum time sustainable in short-circuit. Obviously, doping variation in real

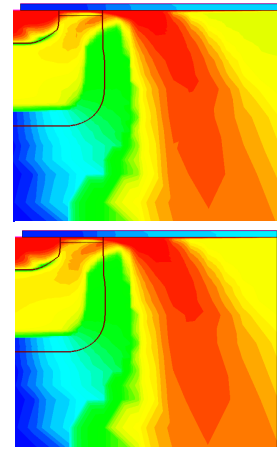

(a)

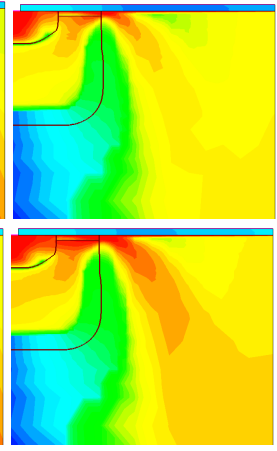

(b)

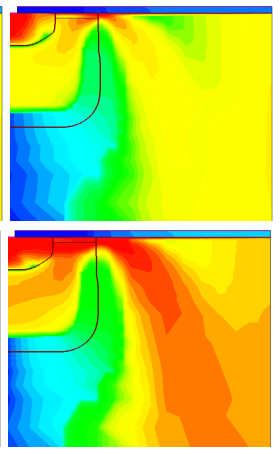

(c)
Fig. 5. Current density for CELL1 (top) and CELL2 (bottom) 


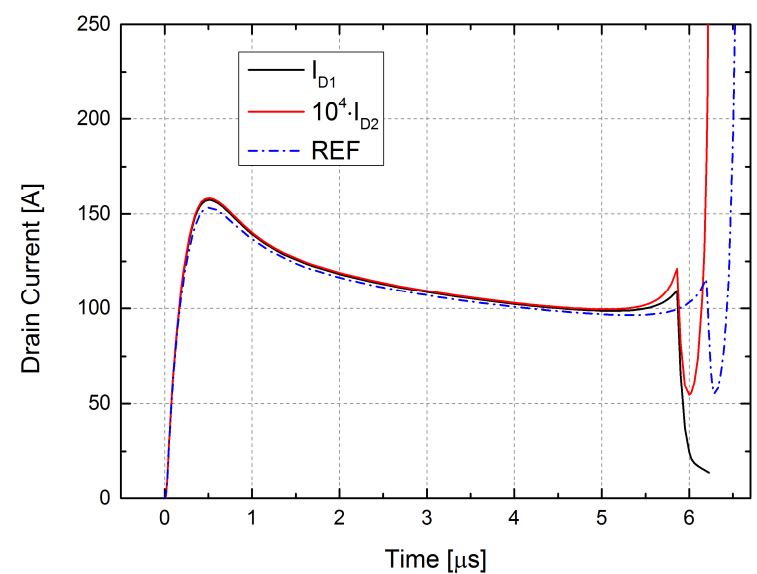

Fig. 6. Simulated $\mathrm{I}_{\mathrm{D}} \mathrm{SC}$ waveforms - channel length mismatch $\left(\mathrm{V}_{\mathrm{DS}}=800 \mathrm{~V} ; \mathrm{V}_{\mathrm{GS}}=18 \mathrm{~V} ; \mathrm{T}_{\mathrm{CASE}}=27^{\circ} \mathrm{C}\right)$

processes are much higher than the value selected, it is therefore clear that areas prone to carry more current could always be present in any device.

Similar result was obtained when the mismatch is introduced in the channel length. Even in this case, an inhomogeneity of $1 \%$ leads to current crowding in a portion of the device (i.e. one of the simulated cells) and to a reduced capability of withstand short-circuit of about 300ns (Fig.6).

Finally, the case of different interface traps concentration was taken into account. Many works reported the significant role given by $\mathrm{SiO}_{2} / \mathrm{SiC}$ interface defects on devices behaviour, due to their impact on mobility and threshold voltage. Importance lies in the correctly modelling their effect [8] and the development of process in order to reduce their density and produce devices with superior performances [12]. Traps concentration could vary within some orders of magnitude depending on the quality of technology process. In this analysis, a change of $2.5 \%$ triggers the failure mechanism about $315 \mathrm{~ns}$ earlier. Current waveforms are depicted in Fig.7, where it is possible to note that traps also slightly modify the dynamics after the turn-off.

A further simulation was performed considering a structure formed by two full cells, where one of the four channels has a

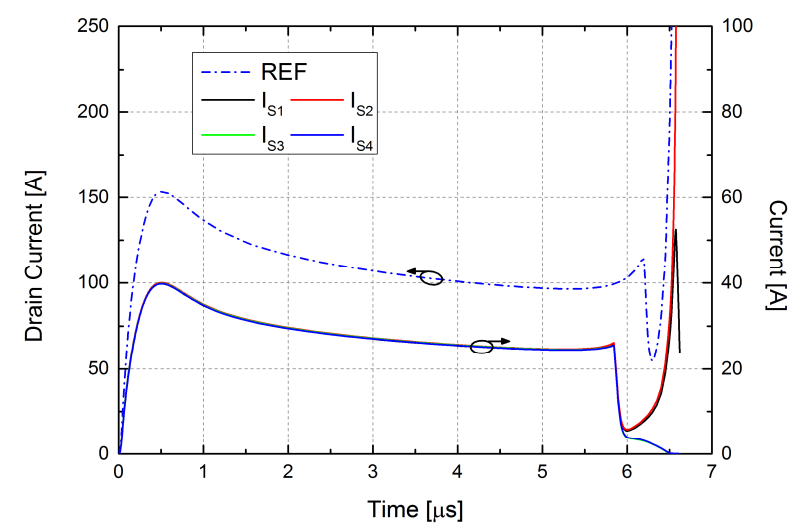

Fig. 8. Simulated current SC waveforms channel doping mismatch, four cells structure $\left(\mathrm{V}_{\mathrm{DS}}=800 \mathrm{~V} ; \mathrm{V}_{\mathrm{GS}}=18 \mathrm{~V} ; \mathrm{T}_{\mathrm{CASE}}=27^{\circ} \mathrm{C}\right)$

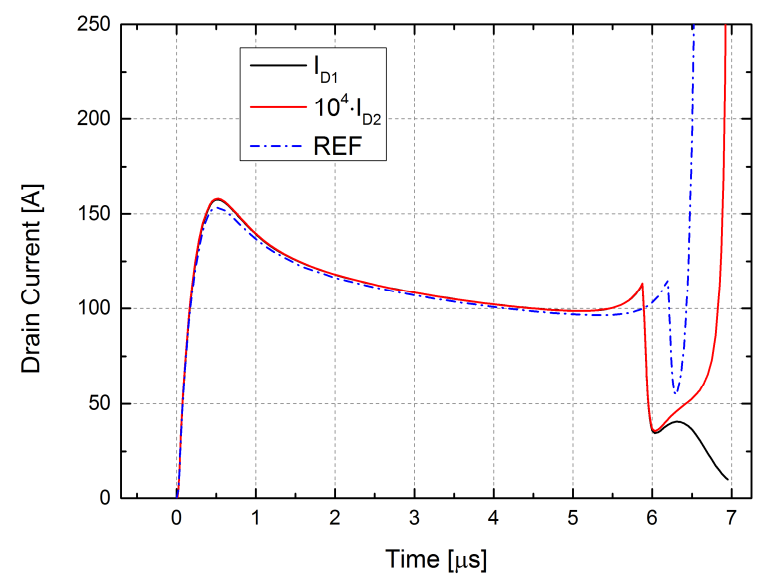

Fig. 7. Simulated $\mathrm{I}_{\mathrm{D}} \mathrm{SC}$ waveforms - interface traps mismatch $\left(\mathrm{V}_{\mathrm{DS}}=800 \mathrm{~V} ; \mathrm{V}_{\mathrm{GS}}=18 \mathrm{~V} ; \mathrm{T}_{\mathrm{CASE}}=27^{\circ} \mathrm{C}\right)$

different doping $(0.75 \%$ smaller peak $)$. The reason is to include, in addition to parameter mismatch, the interaction and the electro-thermal feedback of adjacent cells. Obviously, as the leakage current rises, the weak cell sinks the total device current ( $\mathrm{I}_{\mathrm{S} 2}$ in Fig.8). Fig.9 depicts device current density in different time instants. At turn-off edge (Fig.9a), all four cells have almost the same current value. Slight unbalance appears at the beginning of current tails (Fig.9b), but afterwards just Cell2 exhibits the failure condition (Fig.9c). Moreover, the SC pulse reduction is about $345 \mathrm{~ns}$, which is greater than the previous case with two independent cells. This could be addressed because of the aforementioned electro-thermal interaction, which assists the formation of current filament.

Obtained results are summarised in Tab.1, which reports the mismatch percentage together with the reduction of maximum sustainable SC pulse $\Delta \mathrm{t}_{\mathrm{SC}}$ (compared with the reference cell).

TABLE I.

\begin{tabular}{c|c|c}
\hline \multicolumn{1}{c}{ Parameter } & \multicolumn{1}{c}{ Variation } & $\Delta \mathbf{t}_{\mathrm{SC}}$ \\
\hline Channel Doping & $0.75 \%$ & $280 \mathrm{~ns}$ \\
\hline Channel Length & $1 \%$ & $300 \mathrm{~ns}$ \\
\hline Interface Traps & $2.5 \%$ & $315 \mathrm{~ns}$ \\
\hline $\begin{array}{c}\text { Channel Doping } \\
\text { (four cells structure) }\end{array}$ & $0.75 \%$ & $345 \mathrm{~ns}$ \\
\hline
\end{tabular}

\section{CONCLUSION}

In this work, an analysis on possible design parameters mismatch affecting the short-circuit capability of SiC power MOSFETs has been proposed. The failure identified both experimentally and using TCAD simulation, corresponds to the formation of current filament with subsequent elevated energy density dissipated in a reduced area. Due to the mechanism regenerative nature, the current tends to be crowded where non-uniformity among cells creates a hotter (weak) area. Channel doping and length, amount of interface traps were the analysed parameters, which mostly affect SC 


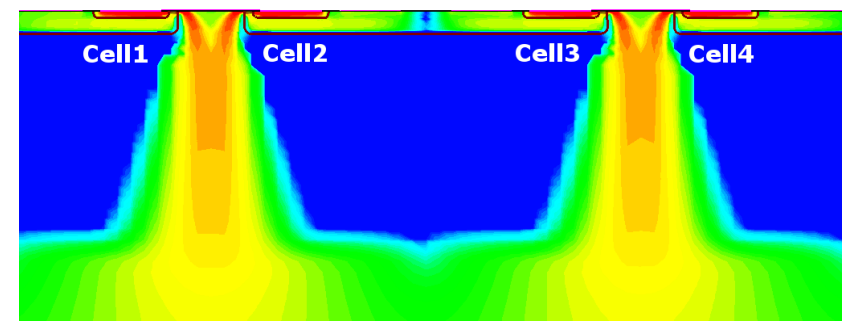

(a) $t=5.8 \mu \mathrm{s}$

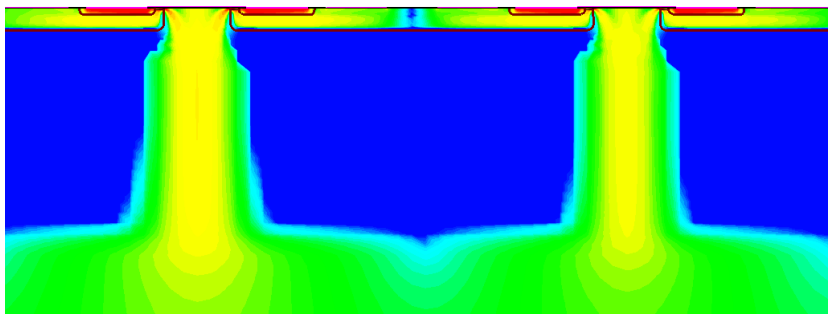

(b) $t=6.1 \mu \mathrm{s}$

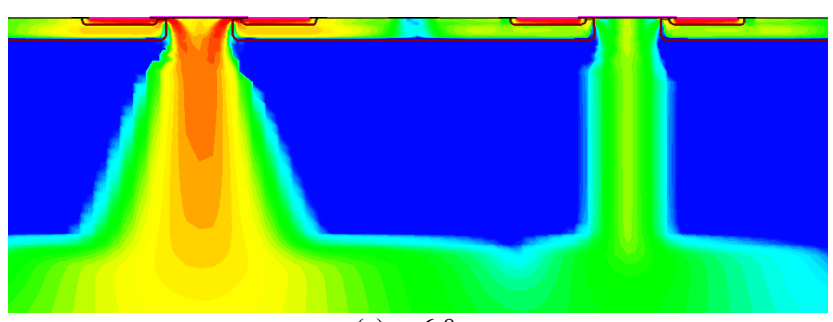

(c) $t=6.8 \mu \mathrm{s}$

Fig. 9. Current density four cells structure $\left(\mathrm{V}_{\mathrm{DS}}=800 \mathrm{~V} ; \mathrm{V}_{\mathrm{GS}}=18 \mathrm{~V} ; \mathrm{T}_{\mathrm{CASE}}=27^{\circ} \mathrm{C}\right)$

behaviour (since they directly act on $\mathrm{V}_{\mathrm{TH}}$ and $\mathrm{R}_{\mathrm{CH}}$ ). As reported, even a minimum percentage difference in the aforementioned parameters (mostly unfeasible for current fabrication technology), leads to hot-spot type failure. Process improvements are of paramount importance to develop devices with better and better performances but an accurate choice of some design strategies should be investigated to increase devices short-circuit reliability.

\section{REFERENCES}

[1] "The Next Generation of Power Conversion Systems Enabled by $\mathrm{SiC}$ Power Devices", ROHM White paper, available online: http://www.rohm.com/documents/11308/f75d744e-efd3-480e-9c96$34 \mathrm{e} 7 \mathrm{db} 3 \mathrm{fad} 9 \mathrm{~d}$
[2] Castellazzi, A.; Fayyaz, A.; Li Yang; Riccio, M.; Irace, A., "Shortcircuit robustness of SiC Power MOSFETs: Experimental analysis", in Power Semiconductor Devices \& IC's (ISPSD), 2014 IEEE 26th International Symposium on, pp.71-74, 15-19 June 2014 doi: 10.1109/ISPSD.2014.6855978

[3] Chen, C.; Labrousse, D.; Lefebvre, S.; Petit, M.; Buttay, C.; Morel, H., "Study of short-circuit robustness of SiC MOSFETs, analysis of the failure modes and comparison with BJTs", in Microelectronics Reliability, vol. 55, issues 9-10, August-September 2015, pp. 17081713

[4] Kampitsis, G.; Papathanassiou, S.; Manias, S., "Comparative evaluation of the short-circuit withstand capability of $1.2 \mathrm{kV}$ silicon carbide (SiC) power transistors in real life applications", in Microelectronics Reliability, vol.55, issue 12, part B, December 2015, pp.2640-2646

[5] Riccio, M.; Castellazzi, A.; De Falco, G.; Irace A., "Experimental analysis of electro-thermal instability in SiC Power MOSFETs", in Microelectronics Reliability, vol 53, issues 9-11, September-November 2013, pp.1739-1744

[6] Shoji, T.; Soeno, A.; Toguchi, H.; Aoi, S.; Watanabe, Y.; Tadano, H., "Theoretical analysis of short-circuit capability of $\mathrm{SiC}$ power MOSFETs", in Japanese Journal of Applied Physics, vol. 54, no. 4S

[7] Romano, G.; Maresca, L.; Riccio, M.; D'Alessandro, V.; Breglio, G.; Irace, A.; Fayyaz, A.; Castellazzi, A., "Short-circuit failure mechanism of SiC power MOSFETs", in Power Semiconductor Devices \& IC's (ISPSD), 2015 IEEE 27th International Symposium on, pp.345-348, 1014 May 2015, doi: 10.1109/ISPSD.2015.7123460

[8] d'Alessandro, V.; Magnani, A.; Riccio, M.; Breglio, G.; Irace, A.; Rinaldi, N.; Castellazzi, A., "SPICE modeling and dynamic electrothermal simulation of $\mathrm{SiC}$ power MOSFETs", in Power Semiconductor Devices \& IC's (ISPSD), 2014 IEEE 26th International Symposium on, pp.285-288, 15-19 June 2014 doi: 10.1109/ISPSD.2014.6856032

[9] S. Potbhare, N. Goldsman, A. Lelis, J. M. McGarrity, F. B. McLean and D. Habersat, "A Physical Model of High Temperature 4H-SiC MOSFETs," in IEEE Transactions on Electron Devices, vol. 55, no. 8, pp. 2029-2040, Aug. 2008

[10] Romano, G.; Riccio, M.; De Falco, G.; Maresca, L.; Irace, A.; Breglio, G., "An ultrafast IR thermography system for transient temperature detection on electronic devices", in Semiconductor Thermal Measurement and Management Symposium (SEMI-THERM), 2014 30th Annual, pp.80-84, 9-13 March 2014 doi: 10.1109/SEMI-THERM.2014.6892219

[11] Kwang-Hoon Oh; Young Chul Kim; Kyu Hyun Lee; Chong Man Yun, "Investigation of short-circuit failure limited by dynamic-avalanche capability in 600-V punchthrough IGBTs", in Device and Materials Reliability, IEEE Transactions on, vol.6, no.1, pp.2-8, March 2006

[12] Uchida, K.; Saitoh, Yu.; Hiyoshi, T.; Masuda, T.; Wada, K.; Tamaso, H.; Hatayama, T.; Hiratsuka, K.; Tsuno, T.; Furumai, M.; Mikamura, Y., "The optimised design and characterization of $1200 \mathrm{~V} / 2.0 \mathrm{~m} \Omega \mathrm{cm}^{2} 4 \mathrm{H}-$ $\mathrm{SiC}$ V-groove trench MOSFETs," in Power Semiconductor Devices \& IC's (ISPSD), 2015 IEEE 27th International Symposium on, pp.85-88, 10-14 May 2015 